\title{
Association of Variants at UMOD with Chronic Kidney Disease and Kidney Stones-Role of Age and Comorbid Diseases
}

\author{
Daniel F. Gudbjartsson ${ }^{1 * 9}$, Hilma Holm ${ }^{19}$, Olafur S. Indridason ${ }^{2}$, Gudmar Thorleifsson ${ }^{1}$, Vidar \\ Edvardsson ${ }^{3,4}$, Patrick Sulem ${ }^{1}$, Femmie de Vegt ${ }^{5}$, Frank C. H. d'Ancona ${ }^{6}$, Martin den Heijer ${ }^{5,7}$, Leifur \\ Franzson $^{8}$, Thorunn Rafnar ${ }^{1}$, Kristleifur Kristjansson ${ }^{1}$, Unnur S. Bjornsdottir ${ }^{4}$, Gudmundur I. Eyjolfsson ${ }^{9}$, \\ Lambertus A. Kiemeney, ${ }^{5,6}$, Augustine Kong ${ }^{1}$, Runolfur Palsson ${ }^{2,4}$, Unnur Thorsteinsdottir ${ }^{1,4}$, Kari \\ Stefansson ${ }^{1,4 *}$
}

1 deCODE genetics, Reykjavik, Iceland, 2 Division of Nephrology, Department of Medicine, Landspitali University Hospital, Reykjavik, Iceland, 3 Children's Medical Center, Landspitali University Hospital, Reykjavik, Iceland, 4 Faculty of Medicine, School of Health Sciences, University of Iceland, Reykjavik, Iceland, $\mathbf{5}$ Department of Epidemiology, Biostatistics, and Health Technology Assessment, Radboud University Nijmegen Medical Centre, Nijmegen, The Netherlands, 6 Department of Urology, Radboud University Nijmegen Medical Centre, Nijmegen, The Netherlands, 7 Department of Endocrinology, Radboud University Nijmegen Medical Centre, Nijmegen, The Netherlands, 8 Department of Genetics and Molecular Medicine, Landspitali University Hospital, Reykjavik, Iceland, 9 The Laboratory in Mjodd, Reykjavik, Iceland

\begin{abstract}
Chronic kidney disease (CKD) is a worldwide public health problem that is associated with substantial morbidity and mortality. To search for sequence variants that associate with CKD, we conducted a genome-wide association study (GWAS) that included a total of 3,203 Icelandic cases and 38,782 controls. We observed an association between CKD and a variant with $80 \%$ population frequency, rs4293393-T, positioned next to the UMOD gene (GenelD: 7369) on chromosome $16 \mathrm{p} 12\left(\mathrm{OR}=1.25, P=4.1 \times 10^{-10}\right)$. This gene encodes uromodulin (Tamm-Horsfall protein), the most abundant protein in mammalian urine. The variant also associates significantly with serum creatinine concentration ( $\mathrm{SCr}$ ) in Icelandic subjects $\left(\mathrm{N}=24,635, P=1.3 \times 10^{-23}\right)$ but not in a smaller set of healthy Dutch controls $(\mathrm{N}=1,819$, $P=0.39$ ). Our findings validate the association between the UMOD variant and both CKD and SCr recently discovered in a large GWAS. In the Icelandic dataset, we demonstrate that the effect on $\mathrm{SCr}$ increases substantially with both age $\left(P=3.0 \times 10^{-17}\right)$ and number of comorbid diseases $(P=0.008)$. The association with CKD is also stronger in the older age groups. These results suggest that the UMOD variant may influence the adaptation of the kidney to age-related risk factors of kidney disease such as hypertension and diabetes. The variant also associates with serum urea $\left(P=1.0 \times 10^{-6}\right)$, uric acid $(P=0.0064)$, and suggestively with gout. In contrast to CKD, the UMOD variant confers protection against kidney stones when studied in 3,617 Icelandic and Dutch kidney stone cases and 43,201 controls (OR=0.88, $P=5.7 \times 10^{-5}$ ).
\end{abstract}

\footnotetext{
Citation: Gudbjartsson DF, Holm H, Indridason OS, Thorleifsson G, Edvardsson V, et al. (2010) Association of Variants at UMOD with Chronic Kidney Disease and Kidney Stones-Role of Age and Comorbid Diseases. PLoS Genet 6(7): e1001039. doi:10.1371/journal.pgen.1001039

Editor: Mark I. McCarthy, University of Oxford, United Kingdom

Received August 25, 2009; Accepted June 23, 2010; Published July 29, 2010

Copyright: (C) 2010 Gudbjartsson et al. This is an open-access article distributed under the terms of the Creative Commons Attribution License, which permits unrestricted use, distribution, and reproduction in any medium, provided the original author and source are credited.

Funding: This study was sponsored by deCODE genetics, Inc. The funders had no role in study design, data collection and analysis, decision to publish, or preparation of the manuscript.

Competing Interests: The authors from deCODE own stocks and stock options in the company.

*E-mail: daniel.gudbjartsson@decode.is (DFG); kstefans@decode.is (KS)

9 These authors contributed equally to this work.
}

\section{Introduction}

Chronic kidney disease (CKD) is a common disorder that can progress to kidney failure and is associated with an increased risk of cardiovascular disease and mortality $[1,2]$. The cause of CKD is not always known and frequently appears multifactorial with hypertension (HTN) and diabetes mellitus (DM) being the most important causes [3-6]. Other causes include intrinsic kidney disorders, atherosclerosis and nephrotoxic drugs $[7,8]$. Studies also indicate a dramatic increase in the prevalence of CKD with advancing age $[9,10]$. With greater lifespan, the burden of CKD is thus steadily rising in the Western world [11], resulting in a substantial impact on the health care system [12].
Previous studies have suggested a genetic contribution to the risk of kidney disease. Heritability estimates of serum creatinine (SCr) and estimated glomerular filtration rate based on SCr (eGFRcrea), both common measures of kidney function, have been reported as $29 \%$ and $33 \%$, respectively [13]. Recently, Köttgen et al. [14] published the first genome-wide association study (GWAS) on eGFRcrea, eGFR based on cystatin C (eGFRcys), another measure of kidney function, and CKD, reporting significant association with eGFRcrea at three loci (UMOD, SHROOM3 (GeneID: 57619) and GATM-SPATA5L1 (GeneIDs: 2628 and 79029)), with eGFRcys at two loci (CST3CST9 (GeneIDs: 1471 and 128822) and STC1 (GeneID: 6781)) and with GKD at one locus $(U M O D)$ [14]. 


\section{Author Summary}

Chronic kidney disease (CKD) is a common condition that is associated with substantial morbidity and mortality and has been recognized as a major public health problem worldwide. Common causes of CKD include hypertension, diabetes, and inflammatory disorders. Previous studies have shown a significant genetic contribution to kidney disease and a recent genome-wide association study yielded a variant in the UMOD gene that affects the risk of CKD. Here, we replicate the association between UMOD and CKD in an independent analysis. We also demonstrate for the first time an interaction between the UMOD variant and age that suggests that this variant may adversely affect the aging kidney and its adaptation to age-related risk factors of kidney disease, such as hypertension and diabetes. Furthermore, we show that the UMOD variant that affects risk of CKD also provides protection against kidney stone disease.

With the aim of discovering sequence variants that associate with kidney function, we conducted a GWAS in a total of 3,203 Icelanders with CKD and 38,782 controls and in 24,635 Icelandic and 1,819 Dutch subjects with SCr information. We found a sequence variant at the $U M O D$ locus that associates with both CKD and SCr at a genome wide-significant (GWS) level, providing an independent replication of the result by Köttgen et al [14]. We also show that this variant interacts with age-related increase in SCr levels with little or no effect on SCr before the age of 50 years, followed by a rapidly growing effect with increasing age. We demonstrate that this variant associates significantly with serum urea, uric acid and suggestively with gout. In contrast to the deleterious effect on kidney function, the variant confers protection against kidney stone disease.

\section{Results/Discussion}

Genome-wide association of variants at the UMOD locus with CKD and SCr

A GWAS of 2.5 million SNPs, either directly genotyped (Illumina HumanHap300 or HumanHapCNV370 bead chips) or imputed based on the HapMap CEU samples [15], was performed on a sample set of 2,903 Icelanders with CKD (see Materials and Methods for sample set description) and 35,818 controls and also on 22,256 Icelandic subjects with SCr information (See QQ-plots in Figure $\mathrm{S} 1$ and Figure S2). The Icelandic SCr measurements came from two laboratories; the Laboratory in Mjodd, a private outpatient laboratory, and the Clinical Biochemistry Laboratory of Landspitali University Hospital (LUH), serving both inpatients and outpatients. These subjects had 5.2 SCr measurements on average (geometric mean) and we used the median $\mathrm{SCr}$ value for each individual in the subsequent analysis. The $\mathrm{SCr}$ values from the two Icelandic laboratories showed similar dependence on age and sex but there was clearly a trend towards higher SCr in the hospital laboratory compared with the outpatient laboratory (Figure S3).

The GWAS on CKD and SCr both yielded several SNPs in high linkage disequilbrium (LD) on chromosome 16p12 with GWS $\left(P<5 \times 10^{-8}\right)$ association to increased risk of $\mathrm{CKD}$ and elevated SCr. For both phenotypes, this signal is tagged by rs4293393-T (Table 1 and Table 2). For CKD, the odds ratio (OR) conferred by rs4293393-T was $1.25(95 \% \mathrm{CI}=1.16-1.34)$ with a corresponding $P$ value of $6.2 \times 10^{-9}$. In an attempt to replicate this finding, rs4293393 was typed in additional 300 Icelandic subjects with CKD and 2,964 controls. The association was nominally significant in the replication sample set and the effect size consistent with the initial observation (Table 1). The combined OR for rs4293393-T in the two Icelandic CKD sample sets was $1.25(95 \% \mathrm{CI}=1.17-1.35)$ and $P=4.1 \times 10^{-10}$. The association between SCr and rs4293393-T on 16p12 was very strong with an effect of $1.86 \mu \mathrm{mol} / \mathrm{L}$ per allele carried and $P=6.7 \times 10^{-20}$ (Table 2). To follow up on these results, we genotyped rs4293393 in 2,379 additional Icelanders with SCr information, significantly replicating the initially observed effect $\left(P=1.4 \times 10^{-5}\right.$, Table 2). Analysis of the combined Icelandic datasets showed a strong GWS association between rs4293393-T and elevated SCr $($ effect $=1.93 \mu \mathrm{mol} / \mathrm{L}$ per allele, $95 \%$ CI $=1.55-2.31 \mu \mathrm{mol} / \mathrm{L}$; $\left.P=1.3 \times 10^{-23}\right)$. Our findings provide an independent replication of the recently reported results by Köttgen et al [14] of an association of this 16p12 locus with CKD and eGFRcrea. The strongest SNP associations outside the UMOD region on chromosome 16p12 are shown in Table S1 (for GKD) and Table S2 (for SCr), respectively.

For further assessment, we tested rs4293393 in 1,819 Dutch subjects with SCr information. These were healthy populationbased controls (see Materials and Methods for sample set description) with $\mathrm{SCr}$ values substantially different from the Icelandic measurements, generally showing lower values and

Table 1. Association of rs4293393-T with chronic kidney disease (CKD).

\begin{tabular}{|c|c|c|c|c|c|c|}
\hline \multirow{2}{*}{$\frac{\text { CKD }}{\text { population }}$} & \multirow{2}{*}{$\frac{\mathbf{N}}{\text { case }}$} & \multirow[b]{2}{*}{ control } & \multicolumn{2}{|c|}{ Frequency } & \multirow[b]{2}{*}{ OR (95\% CI) } & \multirow[b]{2}{*}{$P$} \\
\hline & & & case & control & & \\
\hline Icelandic discovery & 2,903 & 35,818 & 0.831 & 0.798 & $1.25(1.16,1.34)$ & $6.2 \times 10^{-9}$ \\
\hline Icelandic replication & 300 & 2,964 & 0.840 & 0.798 & $1.33(1.05,1.68)$ & 0.019 \\
\hline Icelandic combined & 3,203 & 38,782 & 0.832 & 0.798 & $1.25(1.17,1.35)$ & $4.1 \times 10^{-10}$ \\
\hline$Y O B \geq 1950$ & 143 & 38,782 & 0.811 & 0.798 & $1.09(0.81,1.46)$ & 0.57 \\
\hline $1950>Y O B \geq 1940$ & 355 & 38,782 & 0.821 & 0.798 & $1.16(0.96,1.41)$ & 0.12 \\
\hline $1940>Y O B \geq 1930$ & 1,029 & 38,782 & 0.834 & 0.798 & $1.28(1.14,1.43)$ & $3.1 \cdot 10^{-5}$ \\
\hline $1930>Y O B \geq 1920$ & 1,242 & 38,782 & 0.838 & 0.798 & $1.31(1.18,1.46)$ & $4.1 \cdot 10^{-7}$ \\
\hline $1920>Y O B$ & 434 & 38,782 & 0.825 & 0.798 & $1.19(1.00,1.42)$ & 0.045 \\
\hline
\end{tabular}

Association of rs4293393-T with CKD in Icelandic subjects. Association is shown for the discovery sample set, the replication sample set, the combined Icelandic sample, and finally, for the combined sample stratified by year of birth (YOB) as a proxy for age at onset.

doi:10.1371/journal.pgen.1001039.t001 
Table 2. Association of rs4293393-T with serum creatinine concentration (SCr)

\begin{tabular}{llll}
\hline SCr sample & $\mathbf{N}$ & Effect $(\mathbf{9 5 \%} \mathbf{C l})$ & $\mathbf{P}$ \\
\hline Icelandic discovery & 22,256 & $1.86(1.46,2.25)$ & $6.7 \times 10^{-20}$ \\
Icelandic replication & 2,379 & $2.68(1.47,3.89)$ & $1.4 \times 10^{-5}$ \\
Icelandic combined & 24,635 & $1.93(1.55,2.31)$ & $1.3 \times 10^{-23}$ \\
Dutch replication & 1,819 & $0.38(-0.48,1.25)$ & 0.39 \\
\hline
\end{tabular}

Association of rs4293393-T with SCr in Icelandic and Dutch subjects. Association is shown for the discovery sample set, the Icelandic and Dutch replication sample sets and the combined Icelandic sample. The population frequency of rs4293393-T is 0.80 in Iceland and 0.81 in the Netherlands. Effects are given in $\mu \mathrm{mol} / \mathrm{L}$. doi:10.1371/journal.pgen.1001039.t002

much less variability (Figure S3). Interestingly, no association was observed in the 1,819 healthy Dutch subjects (effect $=0.38 \mu \mathrm{mol} /$ $\mathrm{L}, 95 \% \mathrm{CI}=-0.48-1.25 \mu \mathrm{mol} / \mathrm{L} ; P=0.39)$ (Table 2). Significant heterogeneity was observed between the $\mathrm{SCr}$ association results for the Icelandic and Dutch populations $\left(\mathrm{I}^{2}=90.4 \%, P=0.0013\right)$.

The SNP rs4293393 is located 550 basepairs upstream of UMOD, encoding uromodulin, also known as the Tamm-Horsfall protein (Figure 1). The protein is a glycosylphosphatidylinositol (GPI)-anchored glycoprotein, exclusively expressed in the thick ascending loop of Henle [16] and distal convoluted tubule [17] of the mammalian kidney. It is the most abundant protein in urine of healthy individuals, where it is present in a highly aggregated state $[18,19]$. While the exact physiological function of uromodulin remains to be elucidated, it has the capacity to bind to a variety of ligands. It has been reported to prevent bacteria from adhering to human kidney cells [20] and to inhibit calcium oxalate crystallization [21]. It may also have a role in ion transport and immunological processes [22,23]. UMOD knockout mice have been shown to have decreased creatinine clearance [24] and predilection for both urinary tract infections [25] and calcium oxalate stone formation [26].

The rs4293393 variant is in perfect LD in the HapMap CEU samples [15] with a synonymous SNP in UMOD, rs13335818, coding for $\mathrm{V} 264 \mathrm{~V}\left(\mathrm{D}^{\prime}=1.0, r^{2}=1.0\right)$. The same perfect correlation between rs4293393 and rs13335818 was observed in a set of 3,364 Icelanders $\left(\mathrm{D}^{\prime}=1.0, \quad r^{2}=1.0\right)$. Both $\mathrm{rs} 4293393$ and rs13335818 are also in perfect correlation with rs12917707 $\left(\mathrm{D}^{\prime}=1.0, r^{2}=1.0\right.$ for both markers in the HapMap CEU samples [15]) near the $U M O D$, the variant reported by Köttgen et al [14] to associate with both CKD and eGFRcrea with similar effect, indicating that these SNPs are tagging the same signal. As rs4293393 is on the Illumina $300 / 370 \mathrm{~K}$ chips we used for direct genotyping, we refer to rs4293393 in the remainder of this article.

The effect of the UMOD variant on $\mathrm{SCr}$ is age-dependent

Given that SCr varies substantially with both age and sex, we tested for an interaction between the effect of rs4293393-T and effects of age and sex on SCr. No interaction was found between the $U M O D$ variant and $\operatorname{sex}(P=0.41)$. In contrast, a strong interaction was observed between the $U M O D$ variant and age in the Icelandic sample set $\left(P=3.0 \times 10^{17}\right)$. On average, SCr increased by an additional $0.09 \mu \mathrm{mol} / \mathrm{L}$ per year per allele of rs4293393-T $(95 \%$ CI $=0.07-0.11)$. In order to visualize this interaction, we stratified our Icelandic samples based on age and

\section{$16 \mathrm{p} 12$}

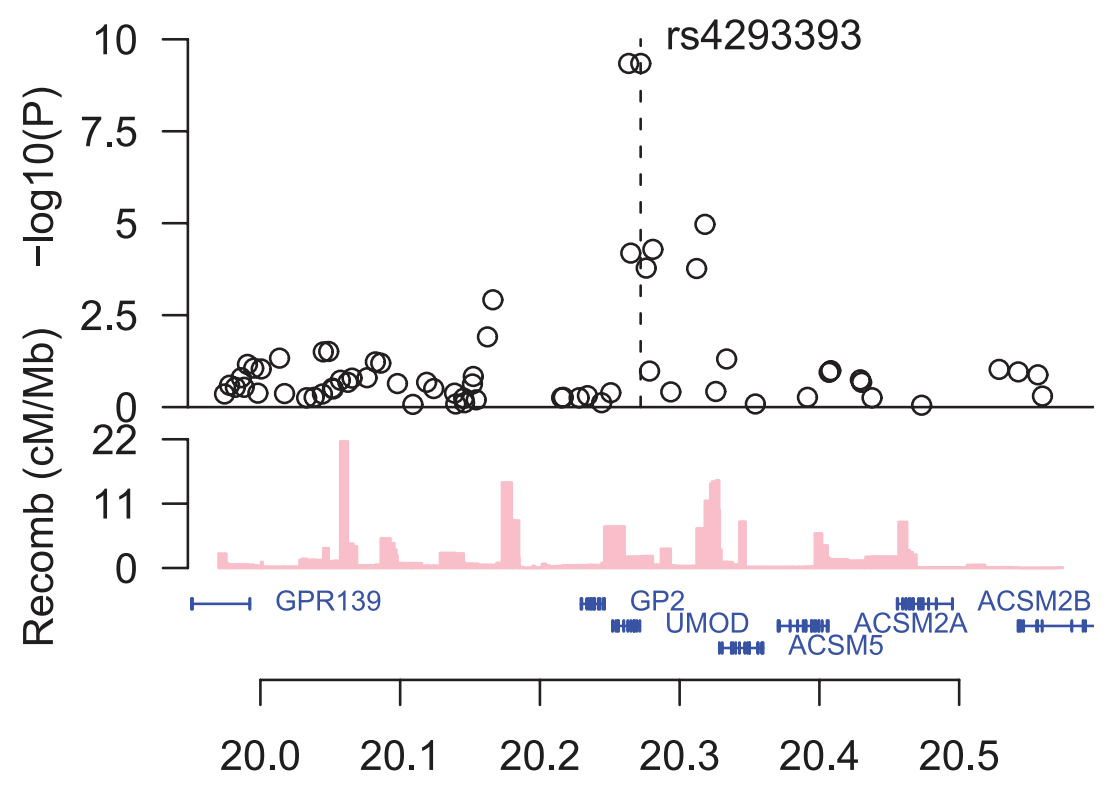

Build 36 position in $\mathrm{Mb}$

Figure 1. An overview of the region around rs4293393. Shown are the $-\log _{10}$ association $P$ values of SNPs in the region with CKD (black circles), the SNPs' build 36 coordinates, the genes in the region and their exons (in blue), and recombination rates in centimorgans (cM) per megabase $(\mathrm{Mb})$ (pink histogram).

doi:10.1371/journal.pgen.1001039.g001 
sex and tested for association within each stratum (Figure 2A). Interestingly, rs4293393-T has little or no effect on SCr before the age of 50 years, but thereafter the effect increases rapidly with advancing age, especially around 70 years. Thus, the variant does not affect $\mathrm{SCr}$ in young individuals but rather how $\mathrm{SCr}$ increases with age. We note that due to the relatively short time span in which the SCr data were collected there is an inherent confounding between age and generation in our study, which will require further investigation to resolve. Similar interaction between the $U M O D$ variant, age and CKD was also observed when the association analysis for CKD was stratified by year of birth used here as a proxy for age of onset (Table 1).

Although it is well known that kidney function declines with age, GFR has been shown to decrease more slowly with senescence in healthy individuals than previously thought $[3-5,11]$. Comorbid conditions that increase in frequency with aging, including HTN, DM, atherosclerosis and heart failure are, however, increasingly recognized as important contributors of age-related decline in kidney function $[3,4,6-8]$. We thus proceeded to investigate whether the age effect observed in carriers of the $U M O D$ variant is influenced by interaction with age-related risk factors for decline in kidney function.

\section{$U M O D$-associated increase in SCr with age is affected by the number of comorbid conditions present}

As HTN, type $2 \mathrm{DM}$ and atherosclerosis are all well recognized age-dependent risk factors for CKD [3,4,6-8], the association analysis was repeated after stratifying the SCr data based on these conditions. Incomplete information regarding history of HTN (5,705 cases), type 2 DM (1,422 cases) and myocardial infarction (MI, 2,551 cases) was available for the Icelandic SCr sample set. In parallel with previous studies, the rate of increase in SCr with age was significantly higher in individuals with HTN than in individuals without this diagnosis (effect $=0.23 \mu \mathrm{mol} / \mathrm{L} /$ year, $95 \% \mathrm{CI}=0.19-0.26 \mu \mathrm{mol} / \mathrm{L} /$ year; $\left.P=2.9 \times 10^{-35}\right)$. Similar results were obtained for type $2 \mathrm{DM}$ (effect $=0.26 \mu \mathrm{mol} / \mathrm{L} /$ year, $95 \%$ CI $=0.19-0.34 \mu \mathrm{mol} / \mathrm{L} /$ year; $\left.P=1.1 \times 10^{-11}\right)$ and MI $($ effect $=0.36 \mu \mathrm{mol} / \mathrm{L} /$ year,$\quad 95 \% \quad \mathrm{CI}=0.30-0.42 \mu \mathrm{mol} / \mathrm{L} /$ year; $\left.P=1.4 \times 10^{-32}\right)$ as well as the number of comorbid conditions (Figure 2B). We also found that the effect of rs4293393-T on SCr increases with the number of comorbid conditions present (Figure 2C).

To further assess the relationship between genotype, age and risk factors for reduced kidney function, we investigated the effect of the rs4293393-T allele count on the increase in SCr with age stratifying on HTN and type 2 DM. A trend was observed for a higher rate of increase in SCr with age and rs4293393-T allele count in individuals with HTN compared to those without a diagnosis of HTN $(P=0.077)$ as well as in those with type $2 \mathrm{DM}$ compared to those without $(P=0.063)$. In other words, the agerelated increase in SCr levels appears to be greater in rs4293393-T carriers that have either HTN or type 2 DM than in carriers who do not have these risk factors. However, an age effect was still observed after accounting for these age-related risk factors. Furthermore, we also observed a significantly higher rate of SCr increase with age and rs4293393-T allele count stratifying on the number of comorbid conditions present $(P=0.0080)$ (Figure 2D).

To determine whether rs4293393-T influenced kidney function by directly affecting known risk factors, we tested the association of rs4293393-T in well powered Icelandic case-control sets of HTN, MI, stroke, and type 2 DM (Table S3). A weak nominally significant association of $\mathrm{rs} 4293393-\mathrm{T}$ with increased risk of HTN was observed $(\mathrm{OR}=1.07,95 \% \mathrm{CI}=1.01-1.12 ; P=0.014)$, but not with the other diseases tested. These data demonstrate that the effect of rs4293393-T on kidney function is not mediated through increased risk of these comorbid conditions, but rather suggest that the variant may affect the vulnerability of the kidney to these risk factors.

These findings, demonstrating not only the effect of age on $U M O D$-associated increase in SCr but also the effect of age-related comorbid conditions, may explain why no association was observed between rs4293393-T and kidney function in the Dutch sample set of healthy population-based subjects with much lower SCr values and of much less variability than observed in the Icelandic samples (Figure S3).

\section{Association of the UMOD variant with serum urea}

Urea is another small solute that is commonly used to assess renal function together with SCr. The correlation between SCr and serum urea in our data was $58 \%$. We tested for association between rs4293393-T and serum urea in an Icelandic sample set that had urea measurements performed at the Laboratory in Mjodd $(\mathrm{N}=4,084)$ and found significant association with increased serum urea concentration (effect $=0.36 \mathrm{mg} / \mathrm{dL}, 95 \% \mathrm{CI}=0.23-$ $\left.0.50 \mathrm{mg} / \mathrm{dL} ; P=1.0 \times 10^{-6}\right)$.

Association of the UMOD variant with uric acid and gout

In humans, rare mutations in the UMOD gene that cause accumulation of abnormal uromodulin in the nephron and reduced urinary excretion of the normal protein [27] have been associated with two autosomal dominant kidney diseases with overlapping clinical features, medullary cystic kidney disease and familial juvenile hyperuricemic nephropathy [28]. These disorders are characterized by hyperuricemia, gout and progressive renal failure due to tubulointerstitial nephropathy. Given the link between $U M O D$ and hyperuricemia, we tested rs4293393-T in Icelandic subjects with serum uric acid values from the Laboratory in Mjodd $(\mathrm{N}=6,583)$ and observed significant association (effect $=6.1,95 \%$ CI $=1.7-10.4 ; P=0.0064)$. We then tested for association with gout in a set of 377 Icelandic cases and 39,916 controls (see Materials and Methods for sample set description) and found a suggestive association $(\mathrm{OR}=1.17,95 \% \mathrm{CI}=0.97-$ 1.41; $P=0.097)$. These data contrast the work of Köttgen et al [14] that neither detected association with serum uric acid nor gout.

\section{Reduced risk of kidney stone formation in carriers of the UMOD CKD risk variant}

As uromodulin is thought to prevent the formation of calciumcontaining kidney stones [21], we tested rs4293393 in a sample set of 1,689 Icelandic patients with radiopaque kidney stones and 37,076 Icelandic population controls. We observed a significant association between rs4293393-T and reduced risk of kidney stones $(\mathrm{OR}=0.88,95 \% \mathrm{CI}=0.81-0.96 ; \quad P=0.0053)$. In an attempt to replicate this observation, we genotyped rs4293393 in two additional sample sets of European ancestry, one from Iceland (1,271 cases and 3,177 controls) and the other from the Netherlands (701 cases and 2,948 controls) (Table 3). The effect size in both replication samples is consistent with the initial observation and the association is significant in the combined replication samples $(\mathrm{OR}=0.89,95 \% \mathrm{CI}=0.81-0.97 ; P=0.0089)$. There was no correlation between the effect size and year of birth of the kidney stone patients (Table S4).

\section{Replication of the SHROOM3 and GATM-SPATA5L1} eGFRcrea loci and the STC1 and CST3-CST9 eGFRcys loci

Köttgen et al [14] reported on variants at additional loci with GWS association to eGFRcrea (SHROOM3 and GATM-SPA- 
A

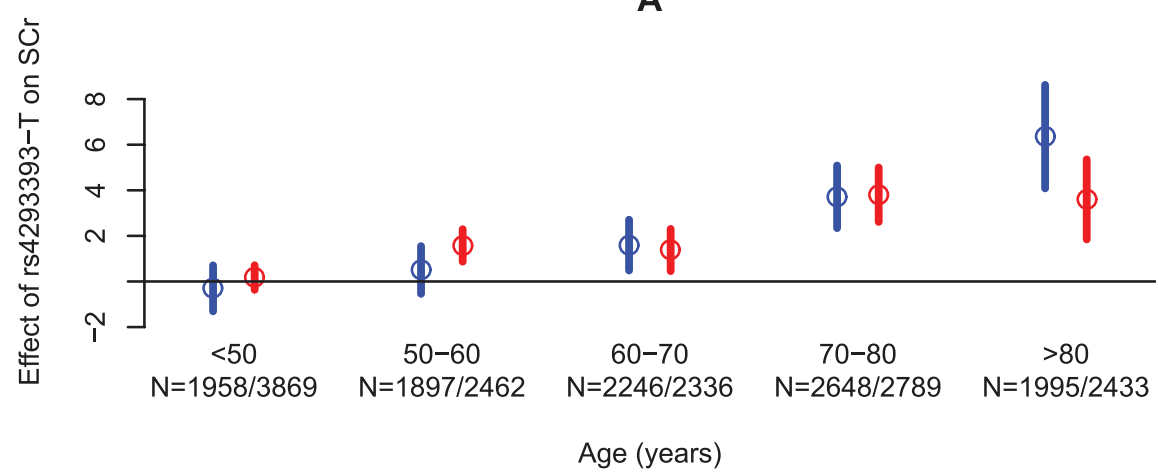

B

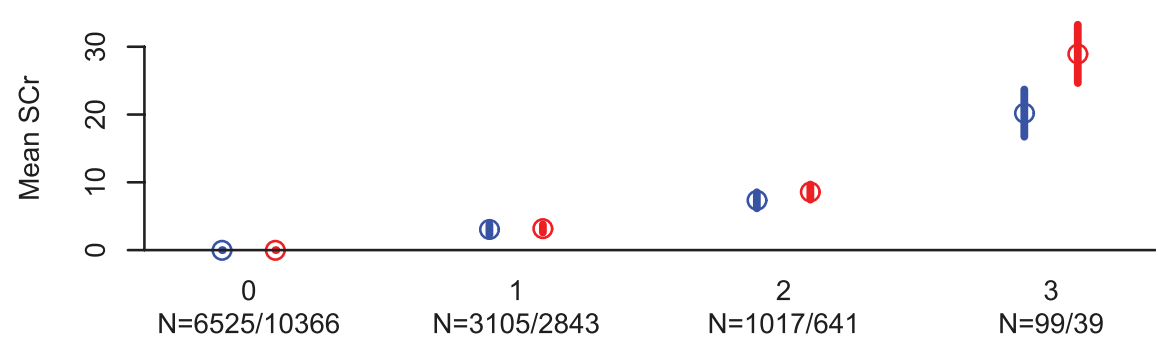

Number of comorbid conditions

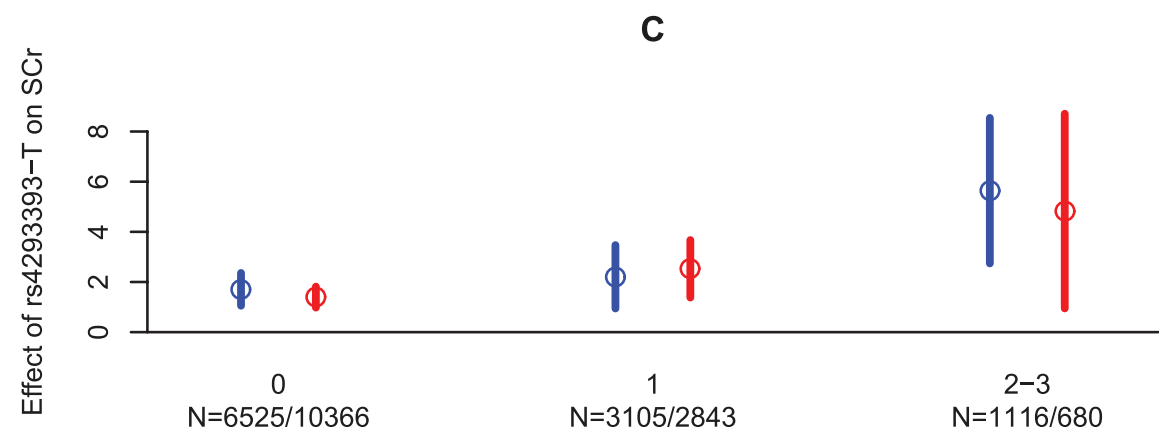

Number of comorbid conditions

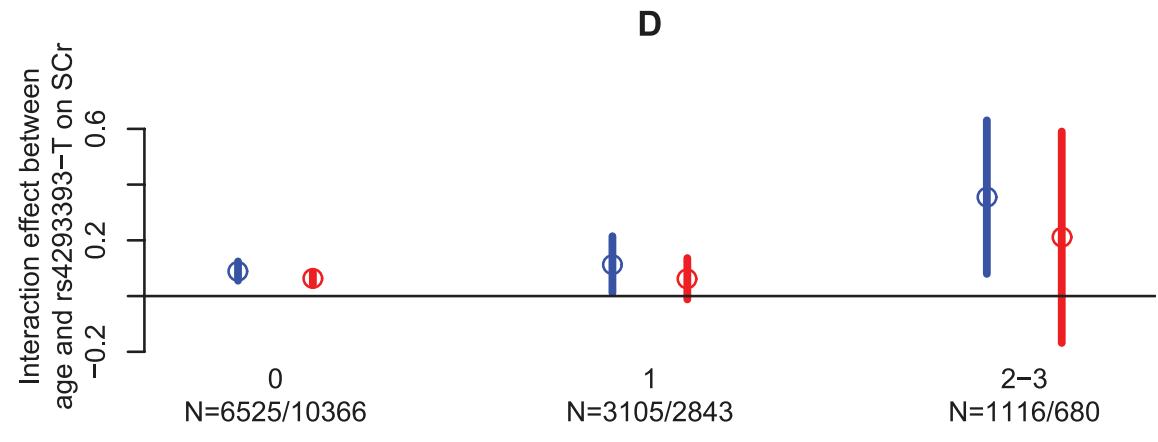

Number of comorbid conditions

Figure 2. An overview of the effect of age and the number of comorbid conditions on SCr levels, directly and through the rs4293393-T allele count. (A) The effect of rs4293393-T on SCr stratified on age and sex. (B) The mean SCr stratified by the number of comorbid conditions and sex, compared to the mean SCr in those without any comorbid conditions. (C) The effect of rs4293393-T on SCr stratified by the 
number of comorbid conditions and sex. (D) The interaction effect between age and rs4293393-T allele count on SCr stratified by the number of comorbid conditions and sex. The circles give the point estimates and the vertical lines show their $95 \%$ confidence intervals. Estimates and confidence intervals are given in blue for males and red for females. Sample sizes (N) are given for each strata for males and females, respectively. Effects are given in $\mu \mathrm{mol} / \mathrm{L}$ in $(A-C)$ and $\mu \mathrm{mol} / \mathrm{L} /$ year in (D).

doi:10.1371/journal.pgen.1001039.g002

TA5L1) or eGFRcys (STC1 and CST3-CST9). We tested these variants in our Icelandic datasets, including a small sample set with cystatin $\mathrm{C}$ measurements (Table 4). The association with SCr replicated for both the SHROOM3 and GATM-SPATA5L1 SNPs $(P=0.00057$ and $P=0.0067$, respectively) and the association with cystatin C replicated for the CST3-CST9 SNP but not the STC1 SNP $(P=0.00037$ and $P=0.73$, respectively). It should be noted, however, that the Icelandic cystatin $\mathrm{C}$ sample set is very small $(\mathrm{N}=284)$ and possibly underpowered to replicate the reported association with the STC1 SNP. The STC1 SNP did show association with SCr in our dataset $\left(P=1.6 \cdot 10^{-6}\right)$ as was observed in the analysis by Köttgen et al [14]. The SHROOM3 and GATMSPATA5L1 SNPs showed suggestive association with CKD in the analysis by Köttgen et al [14] and our data support this association but do not constitute a conclusive replication. In contrast to the $U M O D$ variant, we did not observe an interaction between the variants at these other loci and age. Furthermore, none of the SNPs did associate with kidney stones in Iceland (data not shown). Finally, Köttgen et al [14] reported suggestive association between eGFRcrea and variants at FAG1 (GeneID: 182); we did not replicate this finding in our SCr scan (for rs6040055-T: effect $=-0.27,95 \% \mathrm{CI}=-0.60-0.05), P=0.17)$.

In summary, we describe sequence variants next to and in $U M O D$ that associate with increased risk of CKD and elevated SCr but confer protection against kidney stones. We also demonstrate an interaction between these variants and both age and comorbid conditions that are related to decline in kidney function. Our observations indicate that $U M O D$ is important for maintaining kidney function with advancing age and exposure to risk factors that are associated with aging, such as HTN, type 2 $\mathrm{DM}$ and cardiovascular disease.

\section{Materials and Methods}

\section{Study subjects from Iceland}

Landspitali University Hospital (LUH) is a regional hospital for the greater Reykjavík area and a tertiary referral center for the entire Icelandic nation. The population of Iceland is comprised of 330,000 inhabitants of whom approximately 200,000 reside in the greater Reykjavik area. The nation's only nephrology clinic is located at LUH and all laboratory tests for the primary care clinics of the greater Reykjavik area are performed in the hospital's laboratories. We obtained results of all SCr measurements performed during the period 2003 to 2008 from the computerized database of the Clinical Laboratories at LUH and used the SCr values to identify those with chronic kidney disease (CKD) based on calculation of the estimated glomerular filtration rate (eGFR) by the original 4-variable Modification of Diet in Renal Disease (MDRD) study equation. We classified those with eGFR $<60 \mathrm{ml} /$ $\mathrm{min} / 1.73 \mathrm{~m}^{2}$ as having CKD. All individuals with acute kidney injury and those who had eGFR $<60 \mathrm{ml} / \mathrm{min} / 1.73 \mathrm{~m}^{2}$ of less than 3 months duration were excluded from the GKD sample set. The study included CKD patients that had donated blood through various genetic programs at deCODE genetics.

Biochemical measurements including SCr, serum urea, serum uric acid and serum cystatin $\mathrm{C}$ were available from two laboratories, the Laboratory in Mjodd, Reykjavik, Iceland, a private outpatient laboratory, and the Clinical Biochemistry Laboratory of LUH, serving both inpatients and outpatients. The main referral center for the Laboratory in Mjodd is a multispecialty medical clinic in Reykjavik (Laeknasetrid). The laboratory tests were done in the years 1997-2008 at the Laboratory in Mjodd and in the years 2003-2008 at LUH. The Icelandic SCr measurements came from both laboratories, the Laboratory in Mjodd $(\mathrm{N}=10,260)$ and LUH $(\mathrm{N}=22,898$, of whom 8,523 also had measurements from the Laboratory in Mjodd). At the LUH, the same enzymatic method was used for measurement of SCr during the study period (Vitros 950 Autoanalyzer, Ortho Clinical Diagnostics, Rochester, MN, USA), whereas at the Laboratory in Mjodd, SCr measurements were performed by modified kinetic Jaffe rection assays until May 2007 when an enzymatic method was introduced.

The Icelandic kidney stone cases consisted of patients with confirmed radiopaque kidney stones from the Icelandic Kidney Stone Disease Registry at LUH. The study included kidney stone patients that had donated blood through various genetic programs at deCODE genetics.

The coronary artery disease [29], stroke [30] and type 2 DM $[31,32]$ patient groups from Iceland have been described previously. The HTN sample set includes individuals who have been recruited into various genetic programs at deCODE and have: (1) self-reported HTN; (2) received the diagnosis of HTN at discharge from the LUH; or (3) have attended the Hypertension Clinic at LUH. The gout sample set includes subjects who were

Table 3. Association of rs4293393-T with kidney stones.

\begin{tabular}{|c|c|c|c|c|c|c|}
\hline \multirow{2}{*}{$\begin{array}{l}\text { Kidney stone } \\
\text { population }\end{array}$} & \multirow{2}{*}{$\begin{array}{l}\mathbf{N} \\
\text { case }\end{array}$} & \multirow[b]{2}{*}{ control } & \multicolumn{2}{|c|}{ Frequency } & \multirow[b]{2}{*}{ OR (95\% CI) } & \multirow[b]{2}{*}{$P$} \\
\hline & & & case & control & & \\
\hline Icelandic discovery & 1,689 & 37,076 & 0.781 & 0.801 & $0.88(0.81,0.96)$ & 0.0053 \\
\hline Icelandic replication & 1,271 & 3,177 & 0.786 & 0.801 & $0.91(0.81,1.03)$ & 0.13 \\
\hline Icelandic combined & 2,916 & 40,253 & 0.782 & 0.801 & $0.89(0.83,0.95)$ & 0.00075 \\
\hline Dutch replication & 701 & 2,948 & 0.782 & 0.810 & $0.85(0.73,0.98)$ & 0.022 \\
\hline Combined & 3,617 & 43,201 & - & - & $0.88(0.83,0.94)$ & $5.7 \times 10^{-5}$ \\
\hline
\end{tabular}

Association of rs4293393-T with kidney stone disease in Icelandic and Dutch subjects. Association is shown for the discovery sample set, the Icelandic and Dutch replication sample sets, the combined Icelandic sample, and all the sample sets combined.

doi:10.1371/journal.pgen.1001039.t003 


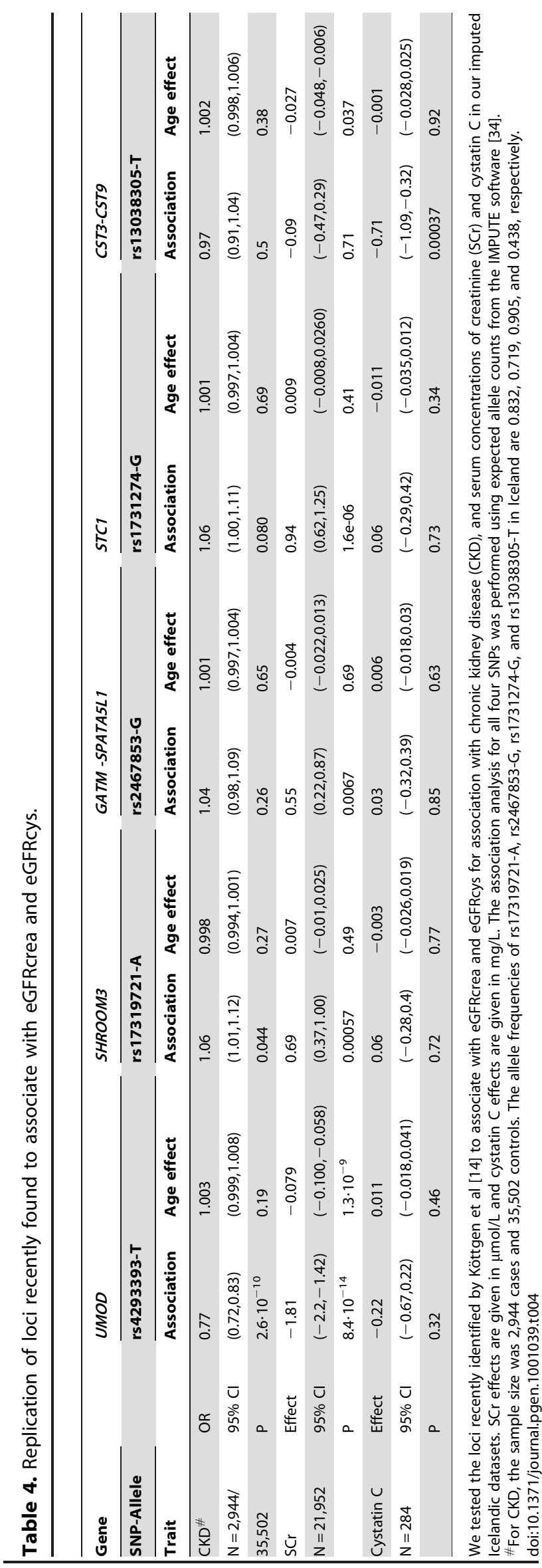

recruited into various genetic programs at deCODE and reported the use of either one of two specific anti-gout medications, allopurinol or colchicine.

The Icelandic controls used in the case-control genome-wide association studies (GWAS) and replication studies were selected among individuals who had participated in the various genetic programs at deCODE genetics; tremor, preeclampsia, endometriosis, psoriasis, type 2 DM, Alzheimer's disease, osteoarthritis, schizophrenia, peripheral artery disease, abdominal aortic aneurysm, chronic obstructive pulmonary disease, stroke, osteoporosis, coronary artery disease, HTN, asthma, Parkinson's disease, sleep apnea, age-related macular degeneration, polycystic ovary syndrome, rheumatoid arthritis, lung cancer, longevity, benign prostatic hyperplasia, enuresis, migraine, glaucoma, attention deficit hyperactivity disorder, prostate cancer, infectious diseases, anxiety, expression studies, autism, dyslexia, melanoma, colorectal cancer, deep vein thrombosis, restless leg syndrome, studies on addiction and population controls. Individuals who reported a history of the trait being tested (e.g. CKD) were excluded from the control set. Some of the controls participated in more than one genetic program in which case their genotypes are only included once.

The study was approved by the Icelandic Data Protection Authority and the National Bioethics Committee. All patients signed informed consent and donated blood samples. Personal identities of the patients and biological samples were encrypted by a third party system provided by the Icelandic Data Protection Authority.

\section{Study subjects from The Netherlands}

All samples with SCr measurements came from the Nijmegen Biomedical Study. The details of this study have been reported previously [33]. Briefly, this is a population-based survey conducted by the Department of Epidemiology and Biostatistics and the Department of Clinical Chemistry of the Radboud University Nijmegen Medical Center (RUNMC), in which 9,371 individuals participated from a total of 22,500 age- and sexstratified, randomly selected inhabitants of Nijmegen. The subjects were invited to participate in a study on gene-environment interactions in complex diseases. All participants filled out a questionnaire on lifestyle and medical history at baseline and 6500 of them donated blood samples for DNA isolation and biochemical studies. A fraction of the study participants were previously genotyped with the Illumina HumanHap300 or HumanHapCNV370 bead chips; these were selected to serve as controls in GWAS on prostate and breast cancer and were selected primarily based on age. A total of 1,819 individuals had both serum creatinine measurements and genome-wide SNP data available for analysis in this study.

The Dutch patients with kidney stones were recruited from two sources: The outpatient clinics of the RUNMC and The Nijmegen Biomedical Study. All patients who present to the outpatient clinics of the RUNMC are invited to participate in a study on the effects of genes and lifestyle on the development of urological diseases. In case of consent, the patients fill out a questionnaire on lifestyle and donate a blood sample for DNA isolation. The controls for the analysis of kidney stone disease were also taken from the biobank of the Nijmegen Biomedical Study. All patients and controls were of self-reported European descent and were fully informed about the goals and the procedures of these studies. The study protocols for the recruitment of patients from outpatient clinics and the recruitment of participants to the Nijmegen Biomedical Study were approved by the RUNMC Institutional Review Board. All study subjects gave written informed consent. 


\section{Illumina genome-wide genotyping}

All Icelandic case and control samples were assayed with the Illumina HumanHap300 or HumanHapCNV370 bead chips (Illumina, SanDiego, CA, USA), containing 317,503 and 370,404 haplotype tagging SNPs derived from phase I of the International HapMap project, respectively. Only SNPs present on both chips were included in the analysis and SNPs were excluded if they had: (a) yield lower than $95 \%$ in cases or controls; (b) minor allele frequency less than $1 \%$ in the population; or (c) showed significant deviation from Hardy-Weinberg equilibrium in the controls $(\mathrm{P}<0.001)$. Any samples with a call rate below $98 \%$ were excluded from the analysis. The final analysis included 302,379 SNPs.

\section{Imputation of SNP genotypes}

The genome-wide association scan was based on expected allele counts obtained with the IMPUTE software [34], using the HapMap CEU samples as a training set [15]. The test for association was then performed using the expected allele counts as covariates. The imputation information was estimated by the ratio of the observed likelihood of allele counts and the likelihood of allele counts assuming perfect information under the assumption of Hardy-Weinberg equilibrium. The estimated information for the four SNPs imputed in Table 4 was high in all instances $(>0.96)$.

\section{Single SNP genotyping}

Single SNP genotyping of all samples was carried out at deCODE genetics in Reykjavik, Iceland, applying the same platform to all populations studied. All single SNP genotyping was carried out using the Centaurus (Nanogen) platform [35]. The quality of each Centaurus SNP assay was evaluated by genotyping each assay on the CEU samples and comparing the results with the HapMap data. All assays had a mismatch rate $<0.5 \%$. Additionally, all markers were re-genotyped on more than $10 \%$ of samples typed with the Illumina platform resulting in an observed mismatch in less than $<0.5 \%$ of samples.

\section{Association analysis}

For case-control association analysis, e.g. for CKD and kidney stones, we utilized a standard likelihood ratio statistic, implemented in the NEMO software [32] to calculate two-sided $P$ values and odds ratios (ORs) for each individual allele, assuming a multiplicative model for risk, i.e. that the risk of the two alleles carried by a person multiplies [36]. Allelic frequencies, rather than carrier frequencies, are presented for the markers and $P$ values are given after adjustment for the relatedness of the subjects. When estimating genotype specific OR, genotype frequencies in the population were estimated assuming HardyWeinberg equilibrium.

Results from multiple case-control groups were combined using a Mantel-Haenszel model [37] in which the groups were allowed to have different population frequencies for alleles, haplotypes and genotypes but were assumed to have common relative risks.

For the quantitative trait association analysis, e.g. for SCr and cystatin $\mathrm{C}$, we utilized a robust linear regression based on an $\mathrm{M}$ estimator [38] as implemented in the rlm function of the $\mathrm{R}$ software package [39]. An additive model for SNP effects was assumed in all instances. All associations with quantitative traits were performed adjusting for age and sex.

\section{Estimation and testing of interaction effects}

Interaction effects were tested by assuming all main effects and lower order interaction effects were present under the null model but not the interaction effect, resulting in a one degree of freedom model. For example, when testing for an interaction effect on SCr between age and the rs4293393-T allele count, the null model included as covariates age, sex and the rs4293393-T allele count. The alternative model included all these covariates as well as the product of the interaction of age and the rs4293393-T allele counts. Similarly, when testing for the interaction between age, the number of comorbid conditions and the rs4293393-T allele count, the null model included as covariates age, sex, rs4293393-T allele count, the product of interaction of age and rs4293393-T allele count, the product of interaction of the number of comorbid conditions and rs4293393-T allele count, and the product of interaction of age and the number of comorbid conditions and the alternative model added the product of interaction of age, the rs4293393-T allele count and the number of comorbid conditions. In the instances when an interaction effect was estimated, the main effect estimates and $P$ values shown were obtained from fitting the appropriate model without an interaction effect.

\section{Correction for relatedness of the subjects and genomic control}

Some of the individuals in the Icelandic patient and control groups are related to each other, causing the chi-square test statistic to have a mean $>1$ and median $>0.675$. We estimated the inflation factor for the genome-wide association by calculating the average of the 302,379 chi-square statistics, which was a method of genomic control [40] to adjust for both relatedness and potential population stratification. The inflation factor was estimated as 1.15 for CKD and 1.22 for SCr and all the results presented from association with these traits were adjusted based on these inflation factors.

\section{Supporting Information}

Figure S1 QQ plot of 2.5 million SNPs in the genome-wide association scans for chronic kidney disease. The black dots represent the observed $\mathrm{P}$ values and the blue ' $\mathrm{x}$ 's represent the $\mathrm{P}$ values scaled down by an inflation factor estimated using genomic control (1.15). The diagonal red line represents where the dots are expected to fall under the null hypothesis of no association. The horizontal green line represents the threshold for genome-wide significance.

Found at: doi:10.1371/journal.pgen.1001039.s001 (0.69 MB TIF)

Figure S2 QQ plot of 2.5 million SNPs in the genome-wide association scans for serum creatinine. The black dots represent the observed $\mathrm{P}$ values and the blue ' $\mathrm{x}$ 's represent the $\mathrm{P}$ values scaled down by an inflation factor estimated using genomic control (1.22). The diagonal red line represents where the dots are expected to fall under the null hypothesis of no association. The horizontal green line represents the threshold for genome-wide significance.

Found at: doi:10.1371/journal.pgen.1001039.s002 (0.69 MB TIF)

Figure S3 The observed distribution of SCr measurements in Iceland and the Netherlands. The measurements from Iceland come from the Laboratory in Mjodd and the Clinical Biochemistry Laboratory of Landspitali University Hospital (LUH). The red line denotes the population median and the two dashed blue lines the $5 \%$ and $95 \%$ quantiles. Measurements above 200 are lumped together for visualization purposes. The unit of measurement is $\mu \mathrm{mol} / \mathrm{L}$.

Found at: doi:10.1371/journal.pgen.1001039.s003 (0.05 MB EPS)

Table S1 Strongest SNP associations $\left(\mathrm{P}<2 \cdot 10^{-5}\right)$ with $\mathrm{CKD}$ outside the $U M O D$ region on chromosome $16 \mathrm{p} 12$. 
Found at: doi:10.1371/journal.pgen.1001039.s004 (0.05 MB DOC)

Table S2 Strongest SNP associations $\left(\mathrm{P}<10^{-6}\right)$ with SCr outside the $U M O D$ region on chromosome $16 \mathrm{p} 12$.

Found at: doi:10.1371/journal.pgen.1001039.s005 (0.10 MB DOG)

Table S3 Association of rs4293393-T with risk factors of kidney function decline in Icelandic case-control groups.

Found at: doi:10.1371/journal.pgen.1001039.s006 (0.03 MB DOC)

Table S4 Results of age-specific association for rs4293393-T and kidney stones using year of birth (YOB) as a proxy for age at onset. Found at: doi:10.1371/journal.pgen.1001039.s007 (0.03 MB DOC)

\section{References}

1. National Kidney Foundation. (2002) K/DOQI clinical practice guidelines for chronic kidney disease: evaluation, classification, and stratification. Am J Kidney Dis 39: $\mathrm{S} 1-266$

2. Sarnak MJ, Levey AS, Schoolwerth AC, Coresh J, Culleton B, et al. (2003) Kidney disease as a risk factor for development of cardiovascular disease: a statement from the American Heart Association Councils on Kidney in Cardiovascular Disease, High Blood Pressure Research, Clinical Cardiology, and Epidemiology and Prevention. Circulation 108: 2154-2169.

3. Lindeman RD, Tobin JD, Shock NW (1984) Association between blood pressure and the rate of decline in renal function with age. Kidney Int 26: 861-868.

4. Fliser D, Franek E, Joest M, Block S, Mutschler E, et al. (1997) Renal function in the elderly: impact of hypertension and cardiac function. Kidney Int 51: 1196-1204.

5. Fliser D, Ritz E (1998) Relationship between hypertension and renal function and its therapeutic implications in the elderly. Gerontology 44: 123-131.

6. Ribstein J, Du Cailar G, Mimran A (2001) Glucose tolerance and age-associated decline in renal function of hypertensive patients. J Hypertens 19: 2257-2264.

7. Kasiske BL (1987) Relationship between vascular disease and age-associated changes in the human kidney. Kidney Int 31: 1153-1159.

8. Bleyer AJ, Shemanski LR, Burke GL, Hansen KJ, Appel RG (2000) Tobacco, hypertension, and vascular disease: risk factors for renal functional decline in an older population. Kidney Int 57: 2072-2079.

9. Viktorsdottir O, Palsson R, Andresdottir MB, Aspelund T, Gudnason V, et al. (2005) Prevalence of chronic kidney disease based on estimated glomerular filtration rate and proteinuria in Icelandic adults. Nephrol Dial Transplant 20: 1799-1807.

10. Coresh J, Selvin E, Stevens LA, Manzi J, Kusek JW, et al. (2007) Prevalence of chronic kidney disease in the United States. JAMA 298: 2038-2047.

11. Lindeman RD, Tobin J, Shock NW (1985) Longitudinal studies on the rate of decline in renal function with age. J Am Geriatr Soc 33: 278-285.

12. Smith DH, Gullion GM, Nichols G, Keith DS, Brown JB (2004) Cost of medical care for chronic kidney disease and comorbidity among enrollees in a large HMO population. J Am Soc Nephrol 15: 1300-1306.

13. Fox CS, Yang Q, Cupples LA, Guo CY, Larson MG, et al. (2004) Genomewide linkage analysis to serum creatinine, GFR, and creatinine clearance in a community-based population: the Framingham Heart Study. J Am Soc Nephrol 15: 2457-2461.

14. Köttgen A, Glazer NL, Dehghan A, Hwang SJ, Katz R, et al. (2009) Multiple loci associated with indices of renal function and chronic kidney disease. Nat Genet 41: 712-717.

15. The International HapMap Consortium (2005) A haplotype map of the human genome. Nature 437: 1299-1320.

16. Bachmann S, Koeppen-Hagemann I, Kriz W (1985) Ultrastructural localization of Tamm-Horsfall glycoprotein (THP) in rat kidney as revealed by protein Agold immunocytochemistry. Histochemistry 83: 531-538.

17. Peach RJ, Day WA, Ellingsen PJ, McGiven AR (1988) Ultrastructural localization of Tamm-Horsfall protein in human kidney using immunogold electron microscopy. Histochem J 20: 156-164

18. Pook MA, Jeremiah S, Scheinman SJ, Povey S, Thakker RV (1993) Localization of the Tamm-Horsfall glycoprotein (uromodulin) gene to chromosome 16p12.316p13.11. Ann Hum Genet 57: 285-290.

19. Devuyst O, Dahan K, Pirson Y (2005) Tamm-Horsfall protein or uromodulin: new ideas about an old molecule. Nephrol Dial Transplant 20: 1290-1294.

20. Leeker A, Kreft B, Sandmann J, Bates J, Wasenauer G, et al. (1997) TammHorsfall protein inhibits binding of S- and P-fimbriated Escherichia coli to human renal tubular epithelial cells. Exp Nephrol 5: 38-46.

\section{Acknowledgments}

The authors would like to thank all the individuals that participated in these studies and whose contribution made this work possible. We also thank our valued colleagues who contributed to the data collection and phenotypic characterization of clinical samples, as well as genotyping and analysis of the genome-wide association data.

\section{Author Contributions}

Conceived and designed the experiments: DFG HH OSI VE LAK RP UT KS. Performed the experiments: OSI VE FdV FCHd MdH LF TR KK USB GIE LAK RP. Analyzed the data: DFG HH GT PS AK. Contributed reagents/materials/analysis tools: DFG VE LF GIE. Wrote the paper: DFG HH OSI GT VE RP UT KS.

21. Hess B, Nakagawa Y, Coe FL (1989) Inhibition of calcium oxalate monohydrate crystal aggregation by urine proteins. Am J Physiol 257: F99-106.

22. Saemann MD, Weichhart T, Zeyda M, Staffler G, Schunn M, et al. (2005) Tamm-Horsfall glycoprotein links innate immune cell activation with adaptive immunity via a Toll-like receptor-4-dependent mechanism. J Clin Invest 115: $468-475$.

23. Yu CL, Tsai CY, Lin WM, Liao TS, Chen HL, et al. (1993) Tamm-Horsfall urinary glycoprotein enhances monokine release and augments lymphocyte proliferation. Immunopharmacology 26: 249-258.

24. Bachmann S, Mutig K, Bates J, Welker P, Geist B, et al. (2005) Renal effects of Tamm-Horsfall protein (uromodulin) deficiency in mice. Am J Physiol Renal Physiol 288: F559-567.

25. Bates JM, Raffi HM, Prasadan K, Mascarenhas R, Laszik Z, et al. (2004) Tamm-Horsfall protein knockout mice are more prone to urinary tract infection: rapid communication. Kidney Int 65: 791-797.

26. Mo L, Huang HY, Zhu XH, Shapiro E, Hasty DL, et al. (2004) Tamm-Horsfall protein is a critical renal defense factor protecting against calcium oxalate crystal formation. Kidney Int 66: 1159-1166.

27. Rampoldi L, Caridi G, Santon D, Boaretto F, Bernascone I, et al. (2003) Allelism of MCKD, FJHN and GCKD caused by impairment of uromodulin export dynamics. Hum Mol Genet 12: 3369-3384.

28. Hart TC, Gorry MC, Hart PS, Woodard AS, Shihabi Z, et al. (2002) Mutations of the UMOD gene are responsible for medullary cystic kidney disease 2 and familial juvenile hyperuricaemic nephropathy. J Med Genet 39: 882-892.

29. Helgadottir A, Thorleifsson G, Manolescu A, Gretarsdottir S, Blondal T, et al. (2007) A common variant on chromosome 9p21 affects the risk of myocardial infarction. Science 316: 1491-1493.

30. Gretarsdottir S, Thorleifsson G, Manolescu A, Styrkarsdottir U, Helgadottir A, et al. (2008) Risk variants for atrial fibrillation on chromosome $4 \mathrm{q} 25$ associate with ischemic stroke. Ann Neurol 64: 402-409.

31. Steinthorsdottir V, Thorleifsson G, Reynisdottir I, Benediktsson R, Jonsdottir T, et al. (2007) A variant in CDKALl influences insulin response and risk of type 2 diabetes. Nat Genet 39: 770-775.

32. Gretarsdottir S, Thorleifsson G, Reynisdottir ST, Manolescu A, Jonsdottir S, et al. (2003) The gene encoding phosphodiesterase 4D confers risk of ischemic stroke. Nat Genet 35: 131-138.

33. Wetzels JF, Kiemeney LA, Swinkels DW, Willems HL, den Heijer M (2007) Age- and gender-specific reference values of estimated GFR in Caucasians: the Nijmegen Biomedical Study. Kidney Int 72: 632-637.

34. Marchini J, Howie B, Myers S, McVean G, Donnelly P (2007) A new multipoint method for genome-wide association studies by imputation of genotypes. Nat Genet 39: 906-913.

35. Kutyavin IV, Milesi D, Belousov Y, Podyminogin M, Vorobiev A, et al. (2006) A novel endonuclease IV post-PCR genotyping system. Nucleic Acids Res 34: e128.

36. Rice JA (1995) Mathematical Statistics and Data Analysis. BelmontCA: Wadsworth Inc.

37. Mantel N, Haenszel W (1959) Statistical aspects of the analysis of data from retrospective studies of disease. J Natl Cancer Inst 22: 719-748.

38. Huber PJ (1981) Robust statistics. New York: Wiley. pp 308.

39. Team RDC (2009) R: A Language for Statistical Computing. Vienna, Austria: R Foundation for Statistical Computing.

40. Devlin B, Roeder K (1999) Genomic control for association studies. Biometrics 55: $997-1004$ 